\title{
Etch Profile Control of High-Aspect Ratio Deep Submicrometer $\alpha$-Si Gate Etch
}

\author{
Hyun-Mog Park, Member, IEEE, Dennis S. Grimard, Jessy W. Grizzle, Fellow, IEEE, and \\ Fred L. Terry, Jr., Senior Member, IEEE
}

\begin{abstract}
The well-acknowledged etch profile drift problem in chip production was investigated with a more accurate means of measuring actual etch thickness to monitor and correct this drift. Using a high-aspect ratio, $0.1-\mu \mathrm{m} \alpha$-Si gate structure, the investigation was specifically focused on the control of transition timing in the critical interval from main etch (ME) to over etch (OE). This required reliable endpoint detection of $\alpha$-Si which was achieved through the development of a method employing a Kalman-Bucy filter with a real-time spectroscopic ellipsometer (RTSE). The robustness of our endpoint detection technique was tested and demonstrated under the actual physical and chemical disturbance environments of the etching process. Application of this endpoint detection technique to the etch of a $0.1-\mu \mathrm{m}$ patterned $\alpha$-Si gate also achieved a significant improvement on the etch profile repeatability.
\end{abstract}

Index Terms-Etch, etch profile, etch rate, Kalman-Bucy filter, process control, semiconductor manufacturing, submicron.

\section{INTRODUCTION}

$\mathbf{T}$ HE DRIVE to miniaturize device feature size has exerted tremendous pressure on research to design and produce smaller chips which are both highly reliable and cost-effective. Though the reduction of chip size is governed by Moore's law, the miniaturization itself has given rise to a whole new set of problems that are roughly inversely proportional to the reduction in chip size. Considerable energy has been focused on improving hardware, materials, processes, and measurements in an attempt to enhance productivity in a highly competitive industry. Among these challenges, the etching process, a fundamental stage in the chip production, has proven to be particularly troublesome, especially in the light of ever tightening production tolerances. Because the etching process is irreversible, developing a highly stable etching process that can be precisely controlled in response to the increasing demands of miniaturization becomes crucial, from both the point of profitability and manufactuability. At present, the decrease in device feature size, and the increase in aspect ratio and wafer size, have made it in-

\footnotetext{
Manuscript received October 31, 2000; revised April 2, 2001. This work was supported in part by the Semiconductor Research Corporation under Contract 97-FC-085, DARPA/AFOSR MURI Center under Contract F49620-95-1-0524, and NIST Advanced Technology Program (ATP) under Contract 70NANB8H4067.

H.-M. Park is with Intel Components Research, Hillsboro, OR 97124-6497 USA (e-mail: hyun-mog.park@intel.com).

D. S. Grimard, J. W. Grizzle, and F. L. Terry, Jr., are with the Electronics Manufacturing and Control Systems Laboratory, Department of Electrical Engineering and Computer Science, University of Michigan, Ann Arbor, MI 48109-2122 USA.

Publisher Item Identifier S 0894-6507(01)06554-X.
}

creasingly difficult to achieve a flawless etch. Increased wafer size is a particular challenge to the production of a highly uniform etch. With the decrease of gate oxide thickness, a highly selective low-damage etch is critical. The current solutions to these etch process problems can be roughly categorized into three areas: 1) process development and optimization; 2) hardware development; and 3) process modeling and control [1]-[8].

In this study, we tackle two critical etch process problems, etch rate drift, and etch profile drift, through real-time closed-loop process control. Etch rate drift can result in significant production delay and cost by making it necessary to run more pilot wafers and preproduction trials to adjust an etch recipe for final production use. Fig. 1 illustrates long-term (over eight months) and short-term (within one hour) $\alpha$-Si etch rate variation observed in a Lam TCP 9400SE plasma etching system.

Despite relatively low runtimes and frequent chamber cleaning, we observed a 29.4-nm etch rate drift $(3 \sigma)$ over eight months of etching. The nominal etch rate was 207 $\mathrm{nm} / \mathrm{min}$. Though the short-term etch rate drift we documented is relatively insignificant compared to the long-term drift, it is, nonetheless, evidence of the need for real-time process control. On the other hand, variations in the etch profile, day-by-day or lot-by-lot, can directly influence device speed to the point of exceeding device tolerance [9], [10]. Moreover, the drive to improve device speed has led to significant reduction of critical dimension (CD) tolerance in gate production. In short, it has become increasingly critical to maintain the consistency of production runs at very tight tolerances. Fig. 2 shows the cross-sectional etch profile of $0.1-\mu \mathrm{m} \alpha$-Si gate structures after 10/75/90 s of breakthrough/main etch/over etch in a Lam TCP 9400SE plasma etching system.

Although two identical samples were etched ten days apart under identical etch conditions, a clear etch profile difference was evident. With the increased production cost in semiconductor manufacturing, unidentified and uncorrected process problems result in significant production cost. Thus, it is critical to study a means for the real-time detection and correction of etch rate and etch profile variations.

In this paper, we propose to control etch profile drift by regulating the transition timing from main etch (ME) to over etch (OE). This is achieved utilizing a real-time spectroscopic ellipsometer (RTSE) in combination with a Kalman-Bucy filter to estimate real-time film thickness. The experimental details will be presented in Section II, together with a description of the plasma etching system, the RTSE, and the sample preparation. Section III details the development of a $0.1-\mu \mathrm{m} \alpha$-Si gate etch 


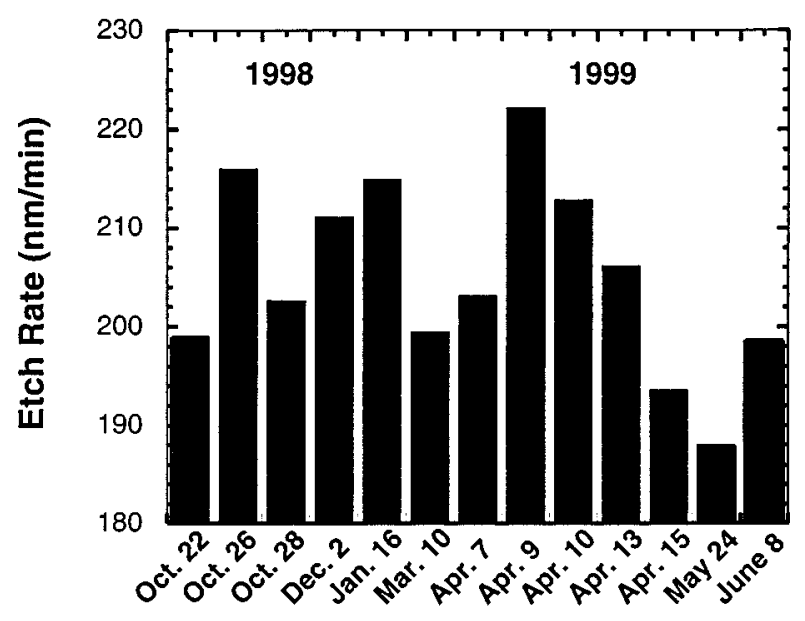

(a) long term



(b) short-term

Fig. 1. Long-term (over eight months) and short-term (within one hour) $\alpha$-Si etch rate drift with the use of a Lam TCP 9400SE plasma etching system.

process and preliminary experimental results of the $0.1-\mu \mathrm{m}$ patterned sample etch. The ME endpoint detection technique developed using a Kalman-Bucy filter is described in Section IV. Finally, Section V examines application of this control method to etch a patterned sample. We conclude with a summary in Section VI.

\section{EXPERIMENTAL DETAILS}

A low-pressure high-plasma density Lam TCP 9400SE plasma etching system with an RTSE were used in our experiment. A brief description of the equipment and a $0.1-\mu \mathrm{m}$ oxide etch mask patterning procedure will be given.

\section{A. Hardware}

1) The Plasma Etching System: Lam TCP 9400SE: For the etch of a deep submicrometer patterned wafer, a low-pressure high-plasma density Lam TCP 9400SE etcher was used (Fig. 3). As a dual-power-source etcher, the TCP 9400SE employs a "transformer coupled" RF power supply to set plasma density

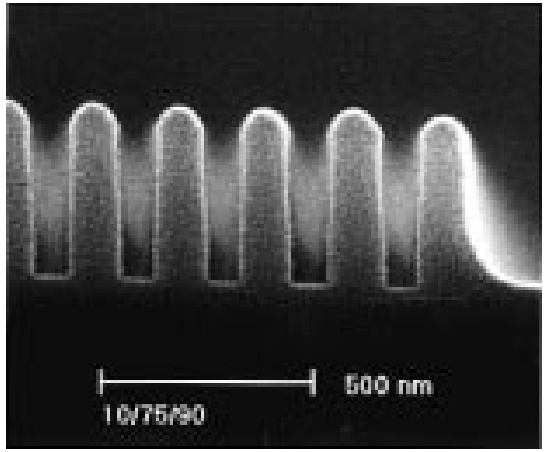

(a)

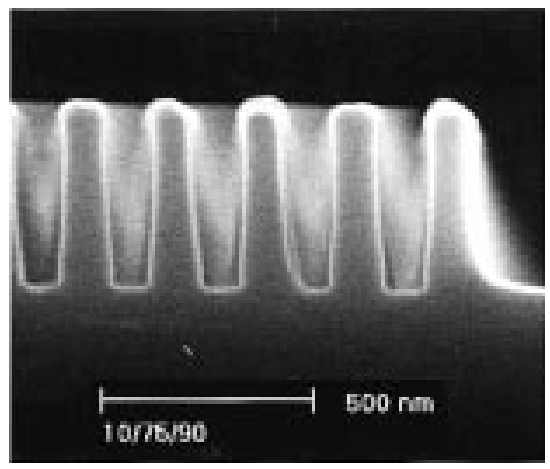

(b)

Fig. 2. Cross-sectional etch profile of $0.1-\mu \mathrm{m} \alpha-\mathrm{Si}$ gate under $10 / 75 / 90 \mathrm{~s}$ of BT/ME/OE in a Lam TCP 9400SE plasma etching system. (a) July 18, 1998. (b) July 27, 1998.

and a standard capacitively coupled RF "bias" power supply to set ion energy. To maximize the delivered power to the plasma reactor and protect the power supplies and cables, a matching network is connected to the power source and electrode. The etcher is equipped with a six-in bipolar electrostatic chuck with helium backside cooling. The temperature controller of the bottom electrode was set to $60{ }^{\circ} \mathrm{C}$ and chamber temperature to $50{ }^{\circ} \mathrm{C}$ throughout the experiment. This plasma etcher is designed for six-in or eight-in wafers with subhalf-micrometer pattern etch of poly-Si, refractory metal silicides, organic antireflection coating (ARC), nitride, and cured photoresist, and thus, $\mathrm{C}_{2} \mathrm{~F}_{6}, \mathrm{Cl}_{2}, \mathrm{H}-\mathrm{Br}, \mathrm{SF}_{6}, \mathrm{O}_{2}, \mathrm{He}$, and $\mathrm{N}_{2}$ gases are supplied. The TCP 9400SE etcher has two built-in photodiode endpoint detectors based on optical emission spectroscopy (OES): 405- and 520-nm wavelength bandpass OES sensors. The 405-nm wavelength matches the $\mathrm{Si}-\mathrm{N}$ and $\mathrm{Al}-\mathrm{Cl}$ emission lines, whereas the 520-nm one matches the $\mathrm{CO}, \mathrm{Si}-\mathrm{Cl}$, and $\mathrm{O}_{2}$ emission lines. To minimize disturbance from the process chamber being exposed to the atmosphere and absorbing water vapor, an entrance and exit loadlock are provided. To acquire data and control the etching process, a PC running LabVIEW was piggybacked onto the Nvision system, which is an original operating system of the Lam TCP 9400SE [11]. A custom LabVIEW-based control program, EMACS, monitors and controls the etching process, while other processes, such as wafer loading/unloading, pumping, and venting, are operated by the Nvision system.

2) Real-Time Spectroscopic Ellipsometer: Ellipsometry is an optical technique that measures changes in the polarization 


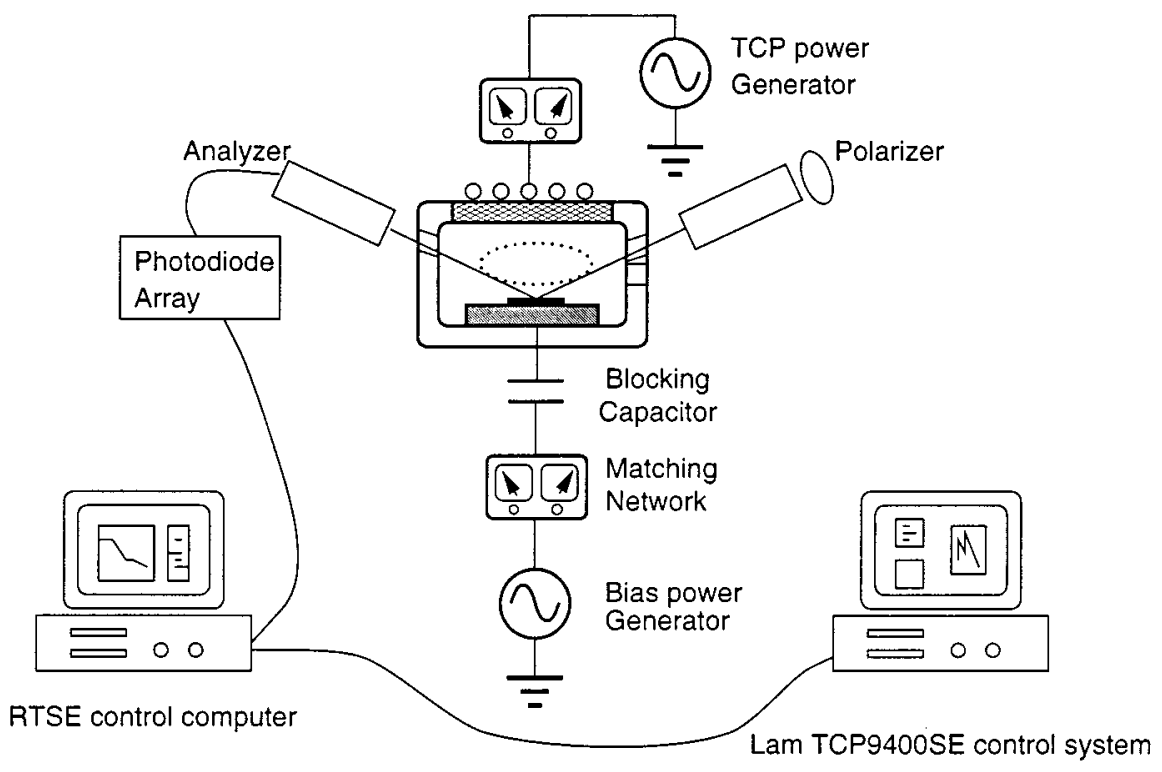

Fig. 3. Schematic of an experimental apparatus.

state of light reflected from a surface. The major advantages of ellipsometry are its nondestructive nature, high sensitivity, large measurement range (from fractions of monolayers to micrometers), and possible real-time implementation. Ellipsometry has been used extensively for in situ film thickness and etch rate measurement [12]-[14], film characterization [15], and in-line monitoring of repetitive patterns [16]. A reflection ellipsometer measures the states of the incident and reflected waves, leading to the determination of the ratio $\rho$ of the complex Fresnel reflection coefficients for the parallel (subscript $p$ ) and perpendicular (subscript $s$ ) polarizations [17]:

$$
\rho=\left(r_{p} / r_{s}\right)=\tan (\Psi) \exp (i \Delta) .
$$

The measured complex ratio is usually expressed in terms of the ellipsometric variables, $\Psi$ and $\Delta$, where $\tan (\Psi)$ corresponds to the attenuation and $\Delta$ to the phase shift between the two components. From these data, the complex index of refraction and film thickness can be determined using a computer model fit. With the assumption that the ambient is air, the reflectance coefficients are directly related to the optical constants of the surface:

$$
r_{p}=\frac{n \cos \phi_{i}-\cos \psi_{i}}{n \cos \phi_{i}+\cos \psi_{i}} r_{s}=\frac{\cos \phi_{i}-n \cos \psi_{i}}{\cos \phi_{i}+n \cos \psi_{i}}
$$

where $n$ is the complex refractive index $n=N-i K$ of the surface.

A commercial RTSE was installed on the Lam TCP 9400SE etching system. For the installation, two small view ports were built in the sidewall of the etch chamber. The light source of the RTSE is a high-pressure Xe arc lamp with an approximate 5-mm beam diameter and intensified photodiode array (IPDA) with controller (a DSP board in a PC) that detects the reflected wave from the surface. The spectral range of the RTSE is $1.8-4.5 \mathrm{eV}$ (approximately 220-560 nm) with 512 pixels for high resolution. The measurement speed is typically $10 \mathrm{~Hz}$ with 10 turns/s for the polarizer. Extraction of the thickness information from the collected ellipsometric data is usually done by the "WinElli" program. However, the "WinElli" is an ex situ film thickness fitting program. Thus, the "FitRTSE" program was used instead, and run with the ellipsometric data collection. This program fits collected ellipsometric data to the optical model of the film under measurement in real time.

\section{B. Sample Preparation}

In order to study deep submicrometer etch, we needed a sample patterned with sufficiently small features. To that end, our samples consisted of a 100-nm-thick oxide etch mask over $200 \mathrm{~nm}$ of phosphorus-doped (n-doped) $\alpha$-Si (respectively, 400 $\mathrm{nm}$ ) on a thermally grown 5-nm-thick gate oxide. $\alpha$-Si was employed for the gate material because its amorphous structure reduces post-etch surface roughness of the gate sidewall. The process flow of the $100-\mathrm{nm}$ oxide etch mask patterning step is illustrated in Fig. 4.

The 100-nm-thick oxide etch mask was patterned using direct exposure electron-beam lithography (EBL) and a liftoff process. A positive e-beam resist, polymethylmethacrylate (PMMA), with a surface-linking promoter, hexamethyldisilazane (HMDS), was spin-coated. To improve the adhesion of the resist and anneal the stresses caused by the shear forces encountered in the spinning process, $30 \mathrm{~min}$ of $180^{\circ} \mathrm{C}$ prebake was followed. After the prebake, the wafer was processed with a 1:3 ratio of methyl isobutyl ketone (MIBK): iso propyl alcohol (IPA) developer for $2 \mathrm{~min}$. This was followed by the electron-beam evaporation of oxide for the hard etch mask patterning. We selected oxide evaporation over thermal oxide growth because of its compatibility with the liftoff process at the expense of its relatively lower etch selectivity over silicon. For future reference, it is worth noting that the cross-sectional profile of the oxide etch mask after the liftoff process is triangular rather than rectangular (Fig. 5).

To investigate the potential effects of microloading during the etching process, both isolated (1:3 and 1:10 line-to-space ratio) 


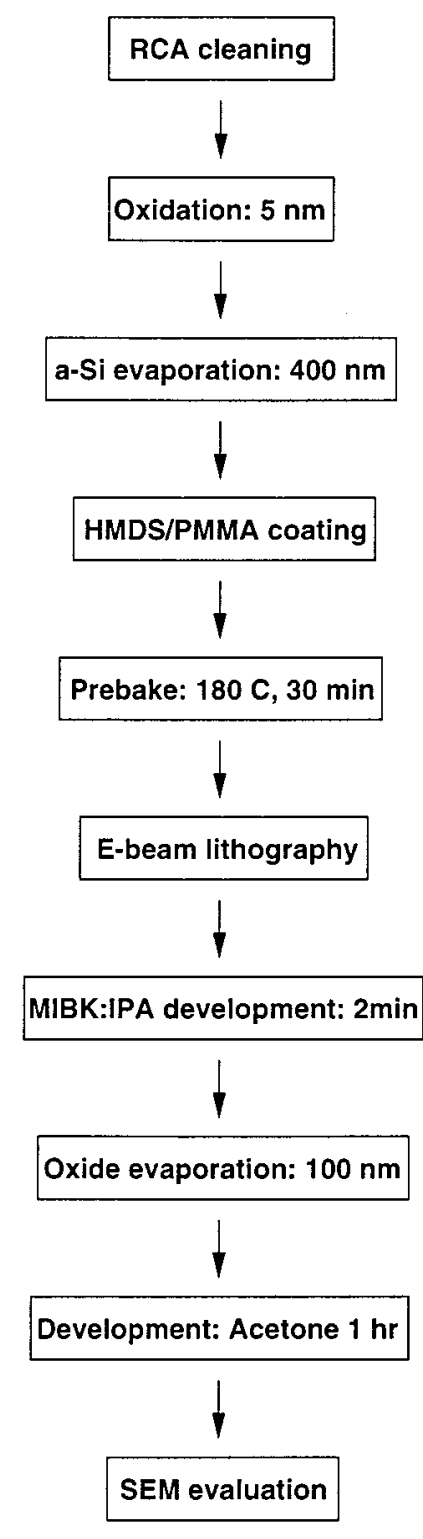

Fig. 4. 100-nm oxide etch mask patterning step employed in this study.

and nested gate-lines (1:1 line-to-space ratio) were patterned on a 4 -in wafer. In this research, a $2 \times 2 \mathrm{~cm}$ patterned sample was used instead of a whole wafer due the well-known problem of long exposure time with EBL. The sample was introduced into the etcher via a carrier wafer: each sample was mounted on a 6 -in silicon wafer via vacuum grease, and then etched. We used a Philips XL 30 FEG scanning electron microscope (SEM) to evaluate the etch profile of the sample after etch.

\section{ETCH PROCESS DEVELOPMENT AND THE IMPORTANCE OF ME TO OE TRANSITION TIMING}

We used a three-step etch recipe consisting of BT, ME, and OE, with chlorine and bromine process gases. The BT step etches a thin layer of native oxide from a wafer surface by employing $\mathrm{C}_{2} \mathrm{~F}_{6}$. Since the subsequent $\mathrm{ME}$ is highly selective to oxide, this BT step is critical in preventing micromasking. To improve etch rate and minimize undercut, a chlorine and bromine gas mix was used in the ME step [1], [2]. The OE step

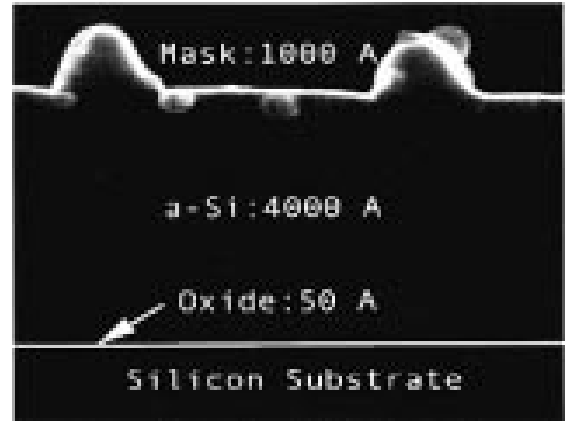

Fig. 5. SEM micrograph of 100-nm-wide and 100-nm-thick oxide etch mask

TABLE I

ETCH RECIPE USED FOR AN n-DOPED $\alpha$-Si GATE-LINE ETCH IN A LAM TCP 9400SE PLASMA ETCHER

\begin{tabular}{l|r|r|r}
\hline Etch step & BT & ME & OE \\
\hline TCP power (W) & 200 & 250 & 300 \\
bias power (W) & 40 & 180 & 200 \\
Pressure (mTorr) & 13 & 10 & 60 \\
$\mathrm{C}_{2} \mathrm{~F}_{6}$ (sccm) & 100 & 0 & 0 \\
$\mathrm{Cl}_{2}(\mathrm{sccm})$ & 0 & 15 & 0 \\
$\mathrm{HBr}(\mathrm{sccm})$ & 0 & 75 & 100 \\
$\mathrm{He}(\mathrm{sccm})$ & 0 & 0 & 200 \\
$\mathrm{O}_{2}(20 \%)+\mathrm{He}(80 \%)(\mathrm{sccm})$ & 0 & 0 & 10 \\
\hline
\end{tabular}

was designed to achieve a very high etch selectivity over oxide (more than 100:1) utilizing mild ion bombardment to minimize gate oxide loss and damage. $\mathrm{HBr}$ was used to maintain a highly anisotropic etch profile in the OE. Since a bromine plasma has a tendency to form localized discharges under reactive ion etching (RIE) plasma condition, helium was added to dilute the $\mathrm{HBr}$ [18]. The noble helium gas has little affect on the surface process that determines the silicon etching characteristics. $\mathrm{O}_{2}$ was also mixed to improve the etch selectivity of $\alpha$-Si with respect to silicon dioxide. The details of the etch recipe are shown in Table I.

The ME and OE recipe were adopted from the Bell Labs recipe [19]; however, our plasma etching system is equipped with a small vacuum pump (1000 $\ell / \mathrm{s})$, forcing us to cut the ME gas flow rate in half. Despite the reduced gas flow, we were only able to maintain a 10 mtorr chamber pressure which is substantially higher than the 2 mtorr of Bell Labs. To increase throughput, the majority of gate material near the gate oxide is etched during the high etch rate ME stage, leaving the residual gate material to be etched under the highly selective OE stage as illustrated in Fig. 6. However, in our experiment, the oxide etch mask was close to triangular since an electron-beam evaporation and liftoff process was used. Because of this etch mask shape, the low etch selectivity of evaporated oxide to $\alpha$-Si, and the high-aspect ratio, the ME had to be terminated when only half of the gate was etched so as to preserve the integrity of the etch mask.

To investigate the effect of ME and OE on the etch profile of our high-aspect ratio gate structure, two sets of experiments were conducted. In the first set, $10 \mathrm{~s}$ of BT was followed by each of 45, 60, and 75 s of ME, without OE step; whereas, in the second set, 10 s of BT was followed by each of 80, 100, and 120 s of OE, with no ME step. To facilitate etch profile comparison, 
TABLE II

CD Measurement of the Two Etch Profiles With Different ME TIME, 45 AND $60 \mathrm{~s}$

\begin{tabular}{l|c|c|c}
\hline & Top & Bottom & Space \\
\hline $10 / 45 / 120 \mathrm{sec}$ & $67 \mathrm{~nm}$ & $125 \mathrm{~nm}$ & $75 \mathrm{~nm}$ \\
$10 / 60 / 120 \mathrm{sec}$ & $55 \mathrm{~nm}$ & $110 \mathrm{~nm}$ & $90 \mathrm{~nm}$ \\
\hline
\end{tabular}

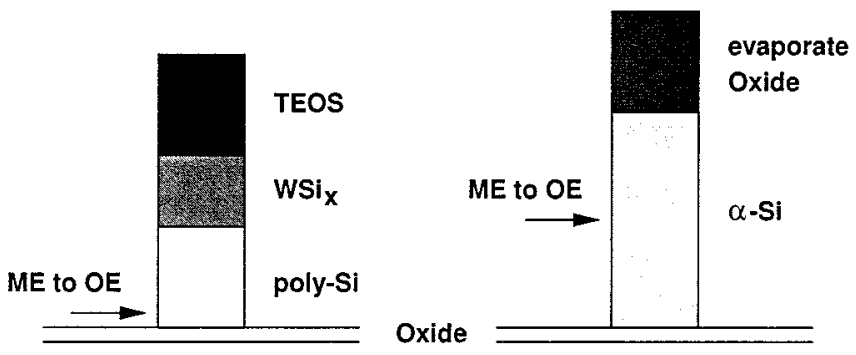

Fig. 6. Gate structures developed in the Bell Labs (left) [19] and the one used in this work (right). They are not drawn in scale.

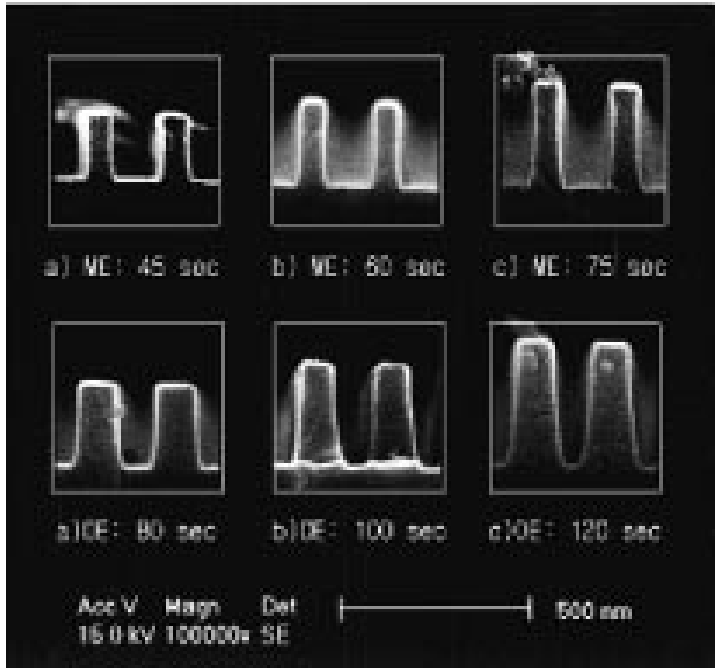

Fig. 7. Cross-sectional etch profile of various ME and OE etch times.

the $\mathrm{OE}$ etch time was set to etch the same etch depth of $\alpha$-Si as in the ME. Fig. 7 illustrates the cross-sectional etch profiles of each experiment.

The samples were dipped in buffered hydrofluoric acid (BHF) solution for $10 \mathrm{~s}$ after the etch to remove any residual oxide etch mask or redeposited oxide-like materials on the sidewall. There was a clear difference observed in the etch profile achieved in the ME with its intensive ion bombardment, compared to that of the $\mathrm{OE}$ with more mild ion bombardment geared to improve etch selectivity. A prolonged ME caused the loss of gate oxide and produced a narrower gate structure due to the loss of etch mask integrity. In the OE, on the other hand, the re-deposition of etch products on the wafer surface with mild ion bombardment contributed to the deterioration of the etch mask pattern transfer. This, in turn, increased the gate CD.

We next examined what affect time variations in the ME would have on the final etch profile. To do so, we conducted the following experiment. After $10 \mathrm{~s}$ of BT to etch the native oxide, one sample was subject to $45 \mathrm{~s}$ of ME, whereas the other sample was subject to $60 \mathrm{~s}$. Then, both samples underwent 120

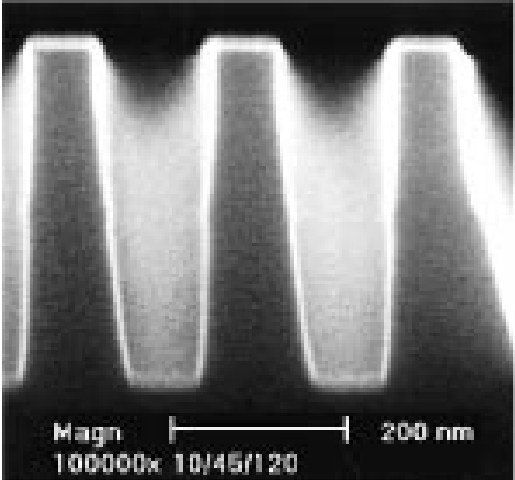

(a)

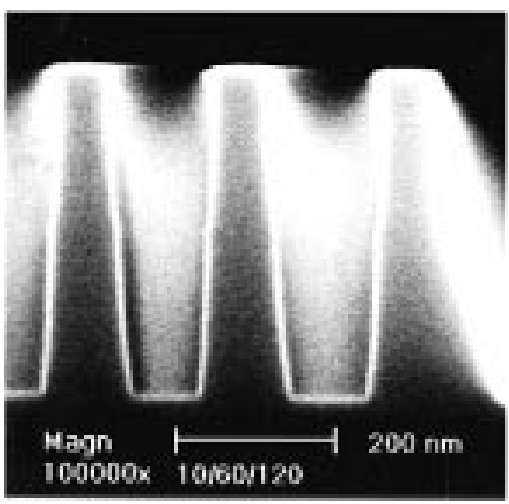

(b)

Fig. 8. Etch profile variation with different ME etch times: $45 \mathrm{~s}$ versus $60 \mathrm{~s}$. (a) 10/45/120 s BT/ME/OE. (b) 10/60/120 s BT/ME/OE.

$\mathrm{s}$ of $\mathrm{OE}$ to etch any residual $\alpha$-Si materials. The cross-sectional etch profiles of the two samples are presented in Fig. 8.

The $60 \mathrm{~s}$ of ME caused greater erosion of the oxide etch mask resulting in a narrower gate top and a wider space between gate structures. At $45 \mathrm{~s}$ of ME, the average $\mathrm{CD}$ for the top of the dense gate lines was $67 \mathrm{~nm}$, as opposed to $55 \mathrm{~nm}$ at $60 \mathrm{~s}$ of ME. In addition, at $45 \mathrm{~s}$ of ME, the space at the foot of the gate between adjacent gate structures proved to be wider than at $60 \mathrm{~s}$ of ME (125 nm as compared to $110 \mathrm{~nm}$ ). It is evident from this experiment that in terms of the amount of gate material etched, the ME is crucial to the final etch profile. Moreover, it becomes obvious that the conventional timed etch in the presence of severe etch rate drift can seriously compromise production consistency.

\section{Development of EndPoint Detection Algorithm}

Once it becomes evident that the transition timing from ME to $\mathrm{OE}$ is a critical stage in maintaining an etch profile, the next task is to develop a system that can accurately determine the transition timing from $\mathrm{ME}$ to $\mathrm{OE}$. This, however, requires measurement of film thickness variation with very fine resolution. To this end, we employed a RTSE system to measure the real-time film thickness of the gate material during the etching process. Unfortunately, due to the limited detection range of the RTSE setup at the University of Michigan, we could not measure the $\alpha$-Si thickness in real time. Therefore, we measured the film thickness of a $2 \times 2 \mathrm{~cm}$ n-doped poly-Si sample placed in the center of the 6-in Si carrier wafer [13]. Assuming constant etch selectivity of poly-Si over $\alpha$-Si, the measured poly-Si thickness 


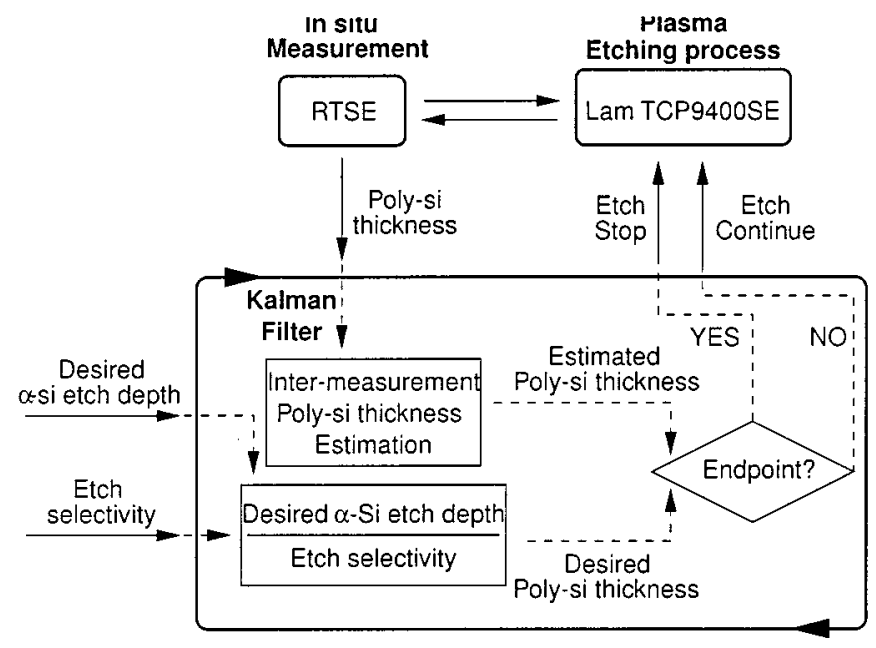

Fig. 9. Diagram of endpoint detection algorithm.

variation was then converted to an equivalent $\alpha$-Si thickness change (Fig. 9).

Although an oxide measurement is much simpler due to its simple optical model, poly-Si was selected at the cost of measurement speed because it has similar etch characteristics to $\alpha$-Si. Our experimental results showed that the long-term oxide to $\alpha$-Si etch selectivity drift $(3 \sigma)$ was approximately $11 \%$ of the average etch selectivity (6.5), whereas n-doped poly-Si to $\alpha$-Si etch selectivity drift was only $2.5 \%$ of the average etch selectivity (0.91). The initial $\alpha$-Si thickness was measured by a SP (Spectra Photometer) before the etch.

The problems which emerged from the use of n-doped poly-Si for the estimation of $\alpha$-Si thickness are slow measurement speed and a time delay coming from the film thickness extraction. The reliable measurement speed of $n$-doped poly-Si in real time, with the concurrent extraction of film thickness from the collected ellipsometrc data, was approximately $0.4 \mathrm{~Hz}$. At the given $0.4-\mathrm{Hz}$ measurement speed, we observed about a 10-nm change of n-doped poly-Si in every measurement under nominal etch conditions. This only provided approximately 9.1 $\mathrm{nm}$ of $\alpha$-Si thickness estimation resolution, assuming constant etch selectivity. Accurate determination of film thickness is further complicated by the time delay inherent in obtaining data from two discrete sources: hardware and software. The RTSE system involves an approximate 0.18 -s time delay to acquire ellipsometric data. This hardware delay reflects the elapsed time in which the reflected light is acquired by the IPDA and transformed into the ellipsometric data format required for the optical model fitting. The software delay, on the other hand, originates from the process of matching the collected ellipsometric data to the optical model of the film layers. The amount of delay depends on the variation of film thickness between measurements and the complexity of the film's optical model. We solved these problems by implementing a Kalman-Bucy filter including a model-based algorithm which compensates for the time delay.

\section{A. Kalman-Bucy Filter Development and Implementation}

The Kalman-Bucy filter is a very efficient, easy to use, recursive least-squares filter. In order to determine the poly-Si thick- ness variation accurately under poor measurement resolution, we built a simple model based on the assumption that the etch rate of poly-Si is a constant, but unknown [20], [21]:

$$
\dot{d}=\text { etch rate }=\text { constant }
$$

where $d$ is the actual poly-Si thickness. Then, the model is

$$
\begin{aligned}
& \ddot{d}=0+v \\
& y=d+w
\end{aligned}
$$

where

$v \quad$ white gaussian noise;

$w$ measurement noise;

$y \quad$ measured poly-Si thickness.

The state equations are given in vector matrix form as

$$
\begin{aligned}
\dot{x}_{t} & =A x_{t}+G v_{t}=\left[\begin{array}{ll}
0 & 1 \\
0 & 0
\end{array}\right] x_{t}+\left[\begin{array}{l}
0 \\
1
\end{array}\right] v_{t} \\
y_{t_{k}} & =C x_{k_{t}}+w_{t_{k}}=\left[\begin{array}{ll}
1 & 0
\end{array}\right] x_{t_{k}}+w_{t_{k}}
\end{aligned}
$$

where subscript $t$ denotes continuous time and $t_{k}$ denotes discrete time. The state vector $x_{t}$ consists of two states, $x_{t}=$ $\left[\begin{array}{ll}x_{1 t} & x_{2 t}\end{array}\right]=[$ film thickness etch rate]. State and measurement noise, $v_{t}$ and $w_{t_{k}}$, are both modeled as independent, zero mean Gaussian with 0.154 variance for $v_{t}$ and 150.7 variance for $w_{t}$. The state equation is a linear continuous-time system as opposed to the output (measurement) equation which is a discrete-time, linear observation taken at time instant $t_{k}$. Between measurements, the optimal (minimum variance) filter for the continuous-discrete system (5) satisfies the differential equations [22]:

$$
\begin{aligned}
\frac{d \hat{x}_{t}^{t}}{d t} & =A \hat{x}_{t}^{t} \\
\frac{d P_{t}^{t}}{d t} & =A P_{t}^{t}+P_{t}^{t} A^{\prime}+G Q G^{\prime}, \quad t_{k} \leq t<t_{k+1}
\end{aligned}
$$

where' denotes matrix transpose. And at measurement $t_{k}$ :

$$
\begin{aligned}
& \hat{x}_{t_{k}}^{t_{k}^{+}}=\hat{x}_{t_{k}}^{t_{k}^{-}}+K\left(t_{k}\right)\left(y_{k}-C \hat{x}_{t_{k}}^{t_{k}^{-}}\right) \\
& P_{t_{k}}^{t_{k}^{+}}=P_{t_{k}}^{t_{k}^{-}}-K\left(t_{k}\right) C P_{t_{k}}^{t_{k}^{-}}
\end{aligned}
$$

with a Kalman-Bucy gain:

$$
K\left(t_{k}\right) \triangleq P_{t_{k}}^{t_{t_{k}}^{-}} C^{\prime}\left[C P_{t_{k}}^{t_{k}^{-}} C^{\prime}+R_{t_{k}}\right]^{-1}
$$

where

$\hat{x}_{t}^{\tau} \quad$ conditional mean of the state $x$ at time $t$ given all measurements $y$ on the interval $[0, \tau]$;

$P \quad$ covariance matrix of states $x$;

$Q \quad$ variance of state noise;

$R \quad$ variance of measurement noise, and $R>0$.

Equation (6) can be approximated by the discrete solution as follows. Between measurements:

$$
\begin{aligned}
\hat{x}_{k+1}^{k} & =\left[\begin{array}{ll}
1 & T \\
0 & 1
\end{array}\right] \hat{x}_{k}^{k} \\
P_{k+1}^{k} & =\left[\begin{array}{ll}
1 & T \\
0 & 1
\end{array}\right] P_{k}^{k}\left[\begin{array}{ll}
1 & 0 \\
T & 1
\end{array}\right]+G Q G^{\prime}
\end{aligned}
$$




\begin{tabular}{llllll} 
& $x$ & & $x$ & 0 & $*$ \\
\cline { 2 - 5 } Time & $\mathrm{t} 1$ & $\mathrm{t} 2$ & $\mathrm{t} 3$ & $\mathrm{t} 4$ & $\mathrm{t} 5$ \\
Thickness & $\mathrm{d} 1$ & $\mathrm{~d} 2$ & $\mathrm{~d} 2$ & $\mathrm{~d} 3$ & $\mathrm{~d} 3$
\end{tabular}

Fig. 10. Illustration of measurement time stamp and thickness information update via RTSE system. $\mathbf{X}$ represents measurement occurrence and $\mathbf{O}$ is when the new measured thickness information is available.

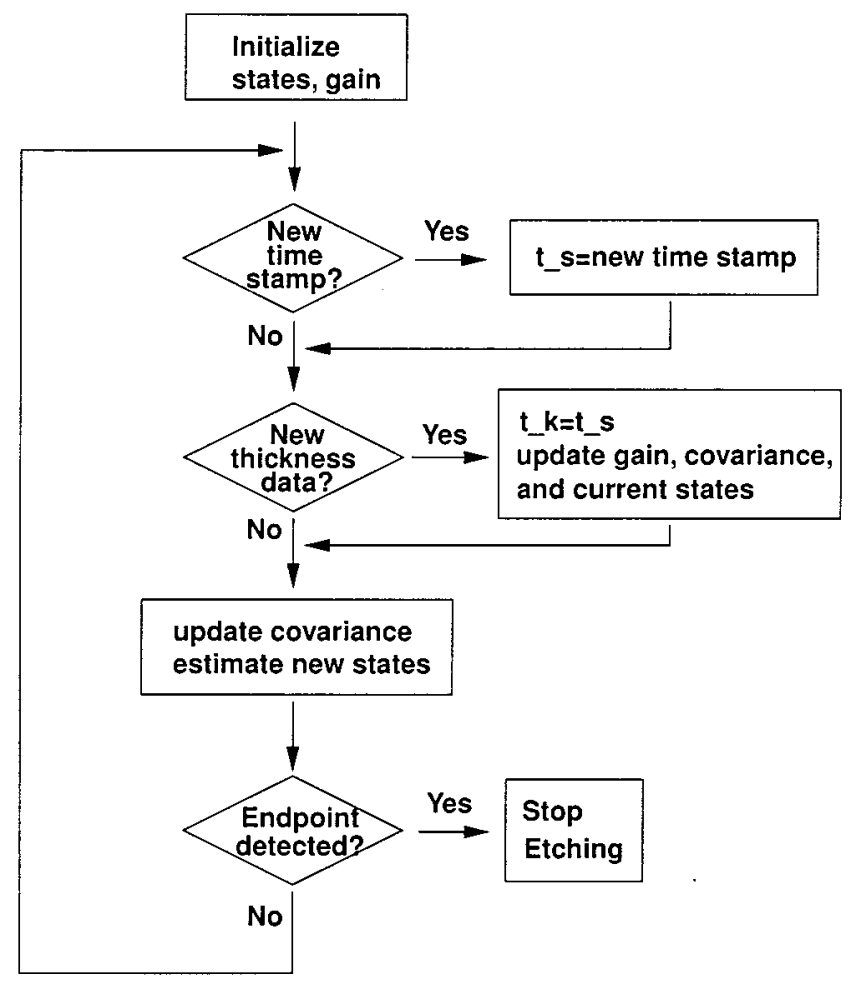

Fig. 11. Flow chart of film thickness estimation based on a Kalman-Bucy filter.

and at measurement:

$$
\begin{aligned}
\hat{x}_{k}^{k} & =\hat{x}_{k}^{k-1}+K(k)\left(y_{k}-C \hat{x}_{k}^{k-1}\right) \\
P_{k}^{k} & =P_{k}^{k-1}-K(k) C P_{k}^{k-1}
\end{aligned}
$$

where $x_{k}$ is the state at $t_{k}, T$ is the time interval between two measurements which was set to $2.5 \mathrm{~s}$ in this experiment, and

$$
K(k)=P_{k}^{k-1} C^{\prime}\left[C P_{k}^{k-1} C^{\prime}+R_{k}\right]^{-1} .
$$

Fig. 10 illustrates when the RTSE measurement occurs and new measured thickness information is available.

If there were no time delay in the thickness extraction from the collected ellipsometric data, new thickness information would be available as soon as the measurement has occurred and could be used immediately to update the Kalman-Bucy gain $(K)$ and covariance matrix $(P)$. However, to compensate for the time delay, we needed to trace two time variables: $t_{s}$, the time when the most recent measurement occurred, and $t_{k}$, the time the most recent Kalman-Bucy gain was updated. As can be seen in Fig. 11, the Kalman-Bucy filter checks the time stamp and thickness information at an estimation interval of $0.1 \mathrm{~s}$. When new film thickness measurement (collecting only ellipsometric data) occurs, the time stamp generated at that same moment is stored in $t_{s}$. Due to the optical model fitting delay, however, new thickness information is updated with a delay of approximately $1.5-1.7 \mathrm{~s}$. When the new thickness information is updated, the Kalman-Bucy gain, covariance matrix $(P)$ and current states are all updated. For instance, in Fig. 10, at time $t_{1}$, a new measurement has occurred, yet, the extracted thickness is only available at time $t_{2}$; the time interval, $t_{2}-t_{1}$, is the elapsed time for the film thickness extraction. When new thickness information $\left(d_{2}\right)$ is available at $t_{2}$, the new thickness $d_{2}$ is compared to the estimated thickness from the Kalman-Bucy filter, and then, the Kalman-Bucy gain and covariance matrix $P$ are adjusted. After the update, the Kalman-Bucy filter continues to estimate the film thickness at a 0.1-s time interval until subsequent thickness information becomes available. This iteration is continued until the endpoint is detected. The Kalman-Bucy filter was implemented as a MATLAB program and tested using actual n-doped poly-Si etch data. After verification of the filter performance, the code was converted into a LabVIEW program running on a PC and used for the endpoint detection of ME. The experimental results are presented in the following section. The MATLAB code is available in the Appendix.

\section{B. Blank Wafer Endpoint Detection Experiment}

1) Vacuum Grease Effect: Due to the difficulty of the mass production of a patterned sample by electron-beam lithography, a small piece of patterned sample was etched. A silicon-based vacuum grease was used to attach a $2 \times 2 \mathrm{~cm}$ patterned sample on a 6-in silicon carrier wafer. The grease serves a dual purpose, providing mechanical stability as well as good thermal conductivity between the sample and the silicon carrier wafer. It is important to note that the etch result for a vacuum grease applied small patterned sample, such as ours, may differ from the etch achieved on a patterned 6-in wafer. Therefore, it is necessary to verify that the experimental result obtained from a small sample with vacuum grease are valid for a 6-in production line wafer. We elected to focus on comparing etch rate since our etch profile control is based on film thickness estimation. Two $\alpha$-Si samples, $2 \times 2 \mathrm{~cm}$ in size, were pasted on a 6-in silicon carrier wafer and etched (Fig. 12). Then, the etch depth of each sample was measured.

We assume that the plasma is uniformly distributed on both samples and that the etch rate difference is only affected by the vacuum grease. The average difference of etch depth between the two samples was $1.35 \mathrm{~nm} / \mathrm{min}$ with a $1.05-\mathrm{nm}$ standard deviation $(\sigma)$. After that, a 6-in $\alpha$-Si wafer was etched under the same etch condition. This wafer had the same film layers as the previously etched $\alpha$-Si sample: $400 \mathrm{~nm}$ of $\alpha$-Si on $5 \mathrm{~nm}$ of gate oxide. The same points were measured as in the previous experiment, and the result showed $1.05-\mathrm{nm} / \mathrm{min}$ etch difference between the two positions with $0.7-\mathrm{nm}$ standard deviation. In terms of the etch nonuniformity, $0.3-\mathrm{nm} / \mathrm{min}$ etch rate variation resulting from the vacuum grease can be considered insignificant compared to the typical etch rate of $207 \mathrm{~nm} / \mathrm{min}$ in the ME. Thus, we conclude that the result of our experiment conducted on a small patterned sample can be transferred to a patterned 6 -in wafer. 


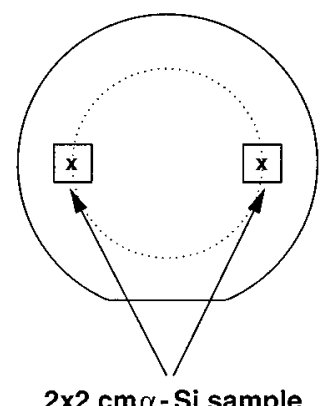
on 6 " Si wafer

Fig. 12. Schematic of the wafers used in the vacuum grease disturbance experiments.

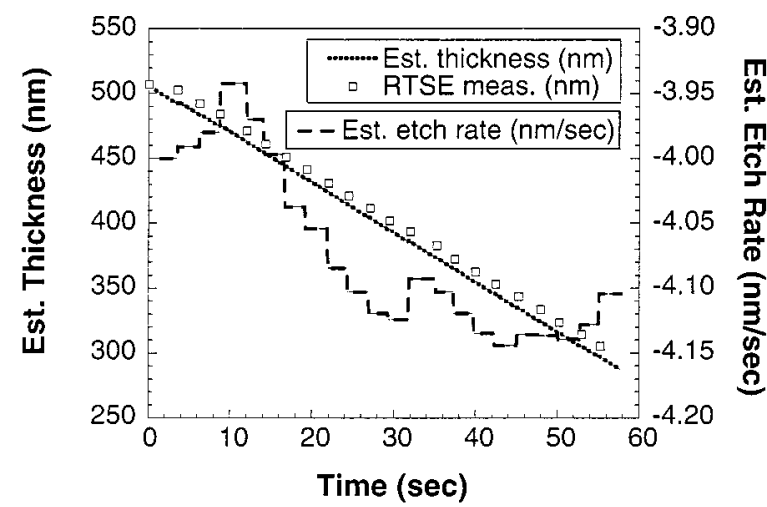

Fig. 13. Experimental result of the poly-Si endpoint detection with the use of a Kalman-Bucy filter.

2) Blank Poly-Si and $\alpha$-Si Endpoint Detection: To determine the performance of the endpoint detection system, two sets of experiments were conducted. In the first experiment, using the Kalman-Bucy filter, we set the endpoint detection system to stop the ME of a blank n-doped poly-Si sample etch when 200 $\mathrm{nm}$ of poly-Si was etched. Fig. 13 illustrates the result of a blank n-doped poly-Si endpoint detection experiment. The square dots are the RTSE measurement of the poly-Si thickness and the dotted line is the estimated thickness at the 0.1-s estimation intervals of the Kalman-Bucy filter. The estimated etch rate fluctuation, shown as a dashed line, is evidence of the well-known oscillations of etch thickness caused by the imperfect reflection index model of poly-Si. The gap between estimated and measured thickness represents a time delay of 1.4-1.7 s, which corresponds to 6-7 $\mathrm{nm}$ difference in thickness. Over repeated tests, we were able to detect the endpoint of blank poly-Si in ME to within $1 \mathrm{~nm}$ accuracy.

The second set of experiments was conducted to determine the accuracy of $\alpha$-Si endpoint detection by observing variation of n-doped poly-Si thickness and estimation of $\alpha$-Si thickness based on the etch selectivity. As shown in Fig. 14, a $2 \times 2 \mathrm{~cm}$ blank $\alpha$-Si sample was placed beside an n-doped poly-Si sample and then etched. The thickness of the $\alpha$-Si sample, before and after etch, was measured by a SP.

The etch results in closed-loop configuration employing an endpoint detection system and open-loop (timed) configuration are compared in Fig. 15. In the closed-loop configuration, the endpoint detection system was programmed to terminate the

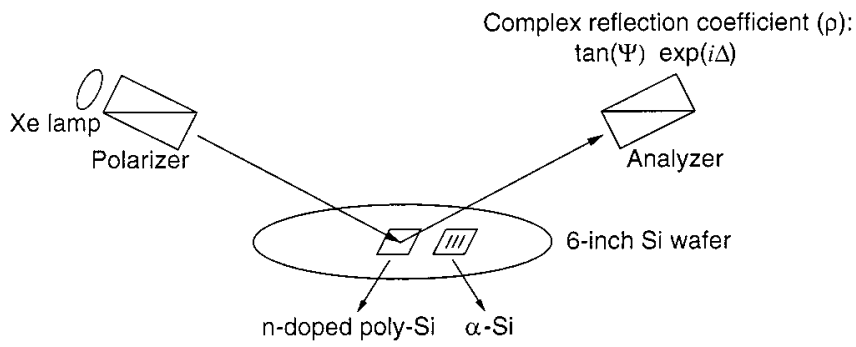

Fig. 14. Schematic of a blank n-doped poly-Si sample etch depth measurement to estimate the $\alpha$-Si etch depth with the use of an RTSE system.

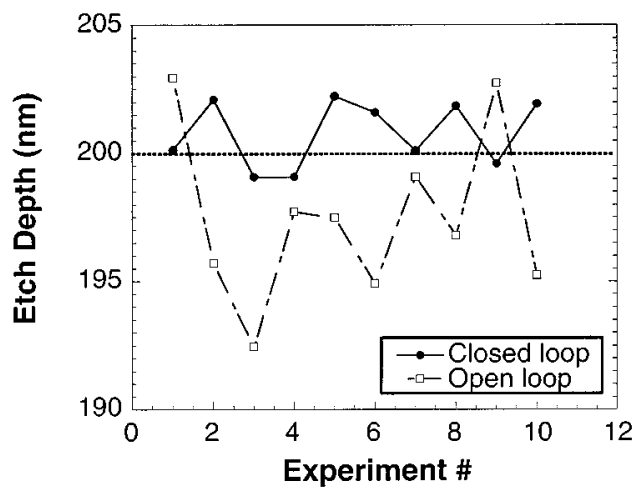

Fig. 15. Comparison of the closed-loop etch and open-loop (timed) etch of $\alpha$-Si.

etch when $200 \mathrm{~nm}$ of $\alpha$-Si was etched. On the other hand, in the open-loop configuration, the ME time was set to $58.2 \mathrm{~s}$ which is the average etch time to etch $200 \mathrm{~nm}$ of $\alpha$-Si under nominal etch conditions. The average etch depth of the closed-loop etch was $200.7 \mathrm{~nm}$ compared to $197.5 \mathrm{~nm}$ of the open-loop etch. Etch depth drift was significantly reduced with the use of endpoint detection system from $10.3 \mathrm{~nm}$ in open-loop etch to $3.9 \mathrm{~nm}$ in closed-loop etch.

\section{Disturbance Rejection}

Though a random disturbance can seriously compromise stability in the etching process and contribute to deterioration of device yield, an intentional disturbance is a useful tool in determining the robustness of a control system. To verify the robustness of our endpoint detection system, we intentionally introduced chemical and physical disturbances into a standard ME. The chemical disturbance consisted of changes in the chemical composition or the concentration of the plasma, whereas the physical disturbance consisted of changes in intensity of ion bombardment energy. In the first experiment employing a chemical disturbance, a chamber was vented and exposed to the atmosphere, and then cleaned with hydrogen peroxide $\left(\mathrm{H}_{2} \mathrm{O}_{2}\right)$ and IPA. In the second experiment, a chemical disturbance was introduced by intentionally changing $\mathrm{Cl}_{2}$ flow rate by $+5 \mathrm{sccm}$. And, in the last experiment, a physical disturbance was introduced by changing bias power by $-15 \%$, decreasing ion bombardment intensity, which in turn decreases the etch rate. In all cases, the endpoint detection system was set to stop the ME when $200 \mathrm{~nm}$ of $\alpha$-Si was etched which takes $58.2 \mathrm{~s}$ under nominal etch conditions. 


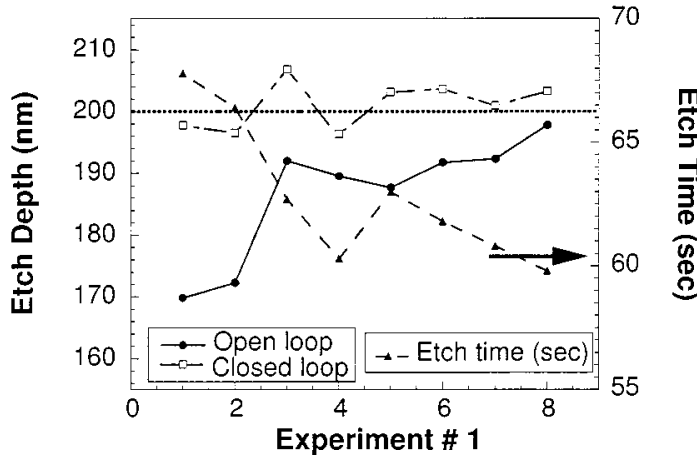

Fig. 16. Comparison of the closed-loop and open-loop (timed) etch under chamber venting disturbance.

The performance of the endpoint detection system in the presence of a chamber venting disturbance (the first experiment) is presented in Fig. 16.

A significant decrease in the etch rate was observed for the first several etches. The absorbed water vapor in the chamber walls evaporates and oxidizes the silicon wafer surface. The extensive oxidation of the $\alpha$-Si surface may retard the etch process resulting in a decreased etch rate. However, as the etch proceeds, the absorbed water vapor becomes exhausted, and therefore, the chamber condition returns to normal. To etch $200 \mathrm{~nm}$ of $\alpha-\mathrm{Si}$, the initial $68 \mathrm{~s}$ of ME was reduced to about $59.5 \mathrm{~s}$ after seven repeated etches. The average closed-loop etch depth was maintained at $201.1 \mathrm{~nm}$ and a $3 \sigma$ of $11.4 \mathrm{~nm}$, as opposed to the open-loop etch depth with an average of $186.7 \mathrm{~nm}$ and a $3 \sigma$ of $30.3 \mathrm{~nm}$. As demonstrated here, the etch process employing an endpoint detection system is obviously superior to the conventional timed etch in its ability to respond to and correct a venting disturbance.

In the second experiment, a chemical disturbance was introduced by increasing the chlorine flow rate by $5 \mathrm{sccm}$. The chamber pressure was kept fixed, forcing the chlorine concentration in the plasma to increase. The high reactivity of chlorine to silicon and the increased proportion of chlorine in the plasma serve to increase the vertical and lateral etch rates. An approximate increase of $6 \%$ in the proportion of chlorine in the total gas flow rate increases the $\alpha$-Si vertical etch rate by $5 \%$. To compensate for the increased etch rate, the endpoint detection system terminated the ME approximately $2.3 \mathrm{~s}$ earlier than the average of $58.2 \mathrm{~s}$ under standard ME conditions (Fig. 17). Although the closed-loop etch provides tighter control of etch depth, it has an etch depth bias of about $7 \mathrm{~nm}$. The etch depth bias may come from the changed etch selectivity of poly-Si over $\alpha$-Si under the chemical disturbance. As mentioned before, the estimation of $\alpha$-Si etch depth is based on the poly-Si etch depth measurement and assumption of constant etch selectivity. The $\alpha$-Si sample used in this experiment has a different crystal structure and phosphorus doping concentration from the n-doped poly-Si sample. Therefore, the etch rate change of $\mathrm{n}$-doped poly-Si may be different from the etch rate of $\alpha$-Si resulting in changed etch selectivity. Thus, the variation of the etch selectivity of n-doped poly-Si over $\alpha$-Si under this chemical disturbance, which was assumed to be a fixed number, may cause the bias.



Fig. 17. Comparison of the closed-loop and open-loop (timed) etch under chlorine flow rate disturbance, $+5 \mathrm{sccm}$.

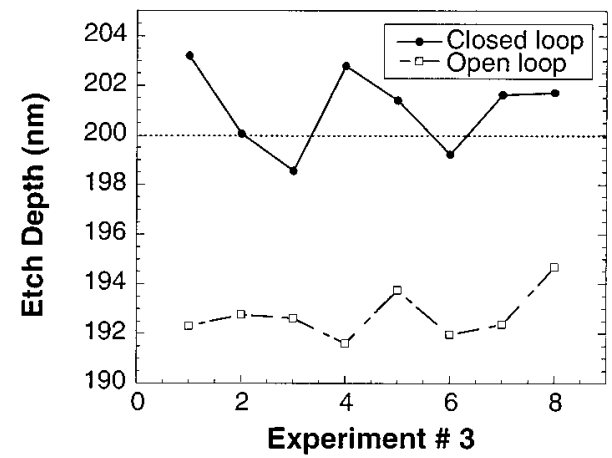

Fig. 18. Comparison of the closed-loop etch and open-loop (timed) etch under bias power disturbance, $-15 \%$.

For the third and last experiment, a physical disturbance was created by decreasing the bias power by $15 \%$ to simulate a power transmission mismatch in a matching network. Decreased bias power reduces the ion bombardment intensity, which reduces the etch rate of both poly-Si and $\alpha$-Si. The effect of decreased bias power on the etch rate is illustrated in Fig. 18. The open-loop etch had an average etch depth of $192.8 \mathrm{~nm}$ for $58.2 \mathrm{~s}$ of ME. However, the closed-loop endpoint detection scheme worked well under the severe bias power disturbance, achieving an average etch depth of $201.1 \mathrm{~nm}$ with a $3 \sigma$ of 4.9 $\mathrm{nm}$. To compensate for the decreased etch rate, the closed-loop endpoint detection system extended the ME time from the typical 58.2 to $60.7 \mathrm{~s}$.

\section{APPLICATION OF ENDPOINT DETECTION}

In the previous section, we presented that a closed-loop etch employing an endpoint detection system with an RTSE and a Kalman-Bucy filter improves the control of etch depth and etch depth variation over the conventional open-loop (timed) etch system, with or without the presence of an intentional disturbance. The previous experiments were performed on blank wafers, yet our goal was to improve the production reliability of an etch profile. We therefore moved on to the etch of patterned samples. First, we used a patterned sample with 100/400/5 nm of an oxide mask $/ \alpha$-Si/gate oxide structure. With this sample 
etch, we intentionally introduced the disturbances described in the previous section. The endpoint detection system was set to terminate the ME when $207 \mathrm{~nm}$ of $\alpha$-Si was etched, then the timed OE continued to etch the rest of the $\alpha$-Si. Next, we cut the $\alpha$-Si thickness to 200 from $400 \mathrm{~nm}$ to simulate industry parameters and etch near to the gate oxide.

\section{A. 0.1-}

We first etched a $0.1-\mu \mathrm{m}$ patterned sample under the standard etch conditions without disturbance: $10 / 60 / 100 \mathrm{~s}$ of BT/ME/OE. A $60 \mathrm{~s}$ of ME etches approximately $207 \mathrm{~nm}$ of $\alpha$-Si under the standard etch conditions. This standard etch profile was used to measure the influence of the disturbance on the etch profile. Then, in the ME, we intentionally introduced a disturbance of a $50 \%$ increase in $\mathrm{Cl}_{2}$ flow rate. In the closed-loop etch, we aimed to stop the ME when the etched $\alpha$-Si etch depth reached 207 $\mathrm{nm}$, and then proceeded to the OE to etch the rest of the $\alpha-\mathrm{Si}$. On the other hand, in the open-loop etch, the patterned sample was etched for 10/60/100 s of BT/ME/OE under the same disturbance as in the closed-loop etch. Fig. 19 illustrates the etch profile of standard, open-loop, and closed-loop etches.

A 15-s dip in BHF was employed to etch any residual oxide etch mask and re-deposited oxide-like materials on the wafer surface. The undercut at the gate bottom was produced during this BHF dip. Due to the increased chlorine flow rate, the lateral etch was enhanced resulting in worsened undercut under the oxide etch mask. In the closed-loop etch, the ME was terminated earlier than $60 \mathrm{~s}$ to compensate for the increased etch rate, and thus it reduced the duration of the lateral etch. The $\mathrm{CD}$ at the gate top was $72 \mathrm{~nm}$ in the standard etch, $48 \mathrm{~nm}$ in the open-loop etch, and $67 \mathrm{~nm}$ in the closed-loop etch. The CD at the gate bottom was $113 \mathrm{~nm}$ in the standard etch, $76 \mathrm{~nm}$ in the open-loop etch, and $106 \mathrm{~nm}$ in the closed-loop etch. It is clearly shown in Fig. 19 that the closed-loop etch profile is more similar to the standard etch than the open-loop etch (see Table III).

\section{B. 0.1- $\mu$ m 200-nm-Thick n-Doped $\alpha$-Si Gate Etch}

In this experiment, we decreased the $\alpha$-Si thickness to 200 $\mathrm{nm}$, aiming to produce a 2:1 aspect ratio gate structure. By changing the gate thickness to $200 \mathrm{~nm}$, we simulated a standard etch condition in the semiconductor manufacturing industry: etch near to a gate oxide in ME. At this juncture, we attempted to etch $190 \mathrm{~nm}$ of 200-nm-thick $\alpha$-Si during the ME, which takes approximately $55 \mathrm{~s}$. A standard etch without any disturbance for 10/55 s of BT/ME was conducted to check any etch profile drift and to provide a baseline. Then, an open-loop etch for 10/55 s of BT/ME was performed with a disturbance in the ME, a $50 \%$ increase in the bias power. And, finally, a closed-loop etch employing endpoint detection was conducted under the same bias power disturbance. With increased bias power, the $\alpha$-Si etch rate was increased because of the intensified ion bombardment. As shown in Fig. 20, the $5 \mathrm{~nm}$ of a gate oxide was fully etched in the open-loop etch, and furthermore, the etch proceeded to the silicon substrate resulting in a 220-nm gate height.

In contrast, the closed-loop etch terminated the ME to compensate for the increased etch rate, resulting in the gate oxide being intact. The closed-loop etch profile under the bias power

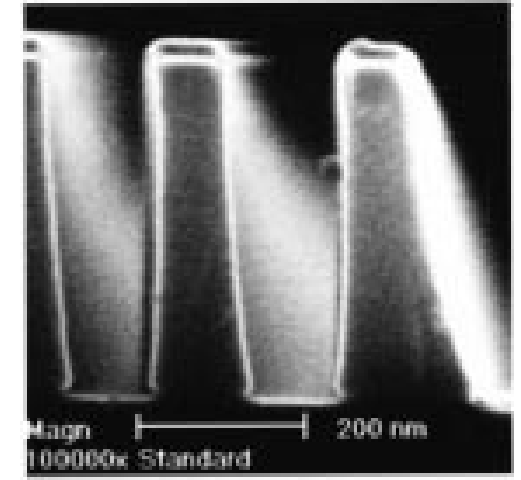

(a)

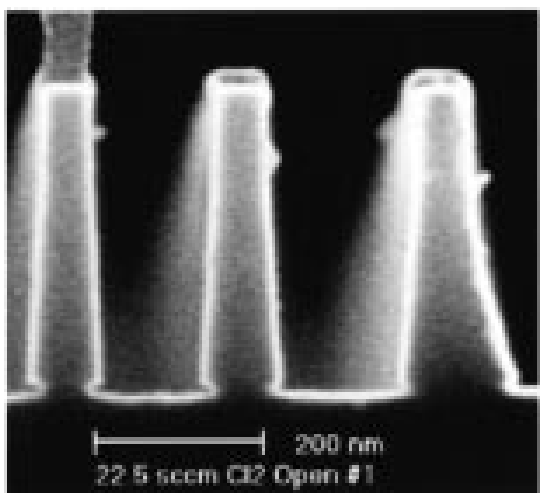

(b)



(c)

Fig. 19. Comparison of the open-loop and closed-loop etch profile under a $50 \%$ increase of $\mathrm{Cl}_{2}$ flow rate disturbance in ME. (a) Standard etch. (b) Open-loop (timed) etch. (c) Closed-loop etch.

TABLE III

MEASURED CD OF STANDARD, OPEN-LOOP, AND ClOSED-LOOP ETCH PROFILE OF 4:1 AND 2:1 ASPECT RATIO GATE ETCH

\begin{tabular}{c|c|c|c}
\hline 4:1 aspect ratio & Standard & Open-loop & Closed-loop \\
\hline Top CD $(\mathrm{nm})$ & $72 \mathrm{~nm}$ & $48 \mathrm{~nm}$ & $67 \mathrm{~nm}$ \\
Bottom CD $(\mathrm{nm})$ & $113 \mathrm{~nm}$ & $76 \mathrm{~nm}$ & $106 \mathrm{~nm}$ \\
\hline \hline 2:1 aspect ratio & Standard & Open-loop & Closed-loop \\
\hline Top CD $(\mathrm{nm})$ & $93 \mathrm{~nm}$ & $54 \mathrm{~nm}$ & $77 \mathrm{~nm}$ \\
Bottom CD $(\mathrm{nm})$ & $108 \mathrm{~nm}$ & $93 \mathrm{~nm}$ & $93 \mathrm{~nm}$ \\
\hline
\end{tabular}

disturbance was comparable to that of the standard etch without disturbance. It should be noted that the rather large bias power increase was employed as a disturbance to demonstrate the etch profile repeatability. That is, under a mild increase in the bias power, an open-loop etch profile would not be as dramatically 


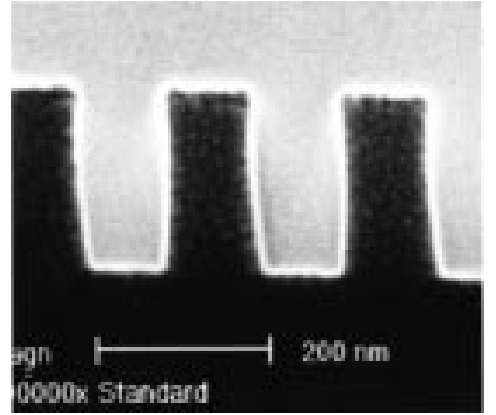

(a)

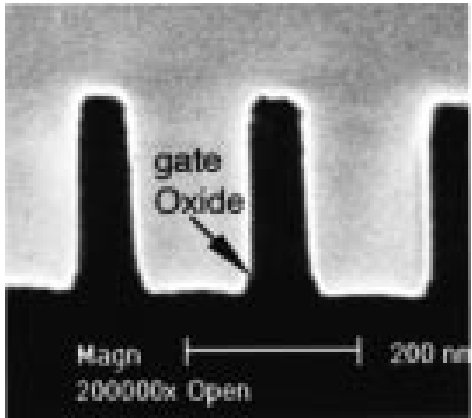

(b)

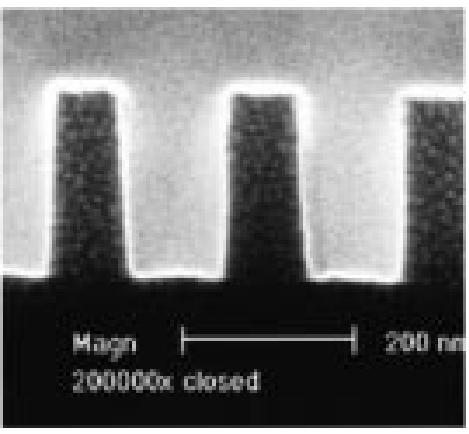

(c)

Fig. 20. Comparison of the open-loop and closed-loop etch profile under a $50 \%$ increase of bias power disturbance in ME. (a) Standard etch. (b) Open-loop (timed) etch. (c) Closed-loop etch.

poor as the one obtained with a $50 \%$ increase. Nonetheless, any possible bias power disturbance in the open-loop etch would adversely affect the gate and silicon substrate by permitting ion penetration through a thinner $\alpha$-Si layer than that of a standard or closed-loop etch. Because of unavailability of the equipment that permits us to evaluate the more subtle influence created by the moderately increased bias power, we were forced to resort to a drastic bias power increase that produced gate oxide loss evident even in visual inspection. The gate width at the top and bottom in the standard etch were 93 and $108 \mathrm{~nm}$, respectively, as opposed to 54 and $93 \mathrm{~nm}$ in the open-loop etch, and 77 and 93 $\mathrm{nm}$ in the closed-loop etch. The narrow gate width has mainly caused by the erosion of the oxide etch mask which was increased by the severe ion bombardment. This is due to the strong dependence of the oxide etch on ion bombardment energy.

To verify the robustness of the developed ME endpoint detection system, a validation experiment was conducted one year later. As a disturbance source, the same amount of $\mathrm{Cl}_{2}$ or bias power disturbance was used to etch 4:1 and 2:1 aspect ratio gate structures, respectively. As shown in Table IV, the closed-loop
TABLE IV

VALIDATION EXPERIMENTAL RESULT OF THE ME ENDPOINT DETECTION of 4:1 AND 2:1 AsPeCt Ratio Gate ETCH UNDER DistuRbanCE

\begin{tabular}{c|c|c|c}
\hline 4:1 aspect ratio & Standard & Open-loop & Closed-loop \\
\hline Top CD $(\mathrm{nm})$ & $84 \mathrm{~nm}$ & $55 \mathrm{~nm}$ & $77 \mathrm{~nm}$ \\
Bottom CD $(\mathrm{nm})$ & $135 \mathrm{~nm}$ & $105 \mathrm{~nm}$ & $124 \mathrm{~nm}$ \\
\hline \hline 2:1 aspect ratio & Standard & Open-loop & Closed-loop \\
\hline Top CD $(\mathrm{nm})$ & $113 \mathrm{~nm}$ & $50 \mathrm{~nm}$ & $90 \mathrm{~nm}$ \\
Bottom CD $(\mathrm{nm})$ & $128 \mathrm{~nm}$ & $108 \mathrm{~nm}$ & $119 \mathrm{~nm}$ \\
\hline
\end{tabular}

etches under disturbance always achieved better etch results than the open-loop etches.

\section{CONCLUSION}

In this paper, we investigated the etch profile control of a high-aspect ratio, $0.1-\mu \mathrm{m} \alpha$-Si gate structure. Since the ME time is critical to the final etch profile, we focused on the detection of the transition timing from ME to OE. For this, an endpoint detection of $\alpha$-Si was developed with the use of a Kalman-Bucy filter and a real-time spectroscopic ellipsometer to observe n-doped poly-Si thickness in real-time. We demonstrated the robustness of our endpoint detection technique under physical and chemical etching disturbance environments. Finally, our endpoint detection system was applied to $0.1-\mu \mathrm{m}$ patterned $\alpha$-Si gate etch. It was proven that the accurate transition timing control from ME to OE improves the etch profile reproducibility.

\section{APPENDIX I}

$M$-FILE FOR ENDPOINT DETECTION

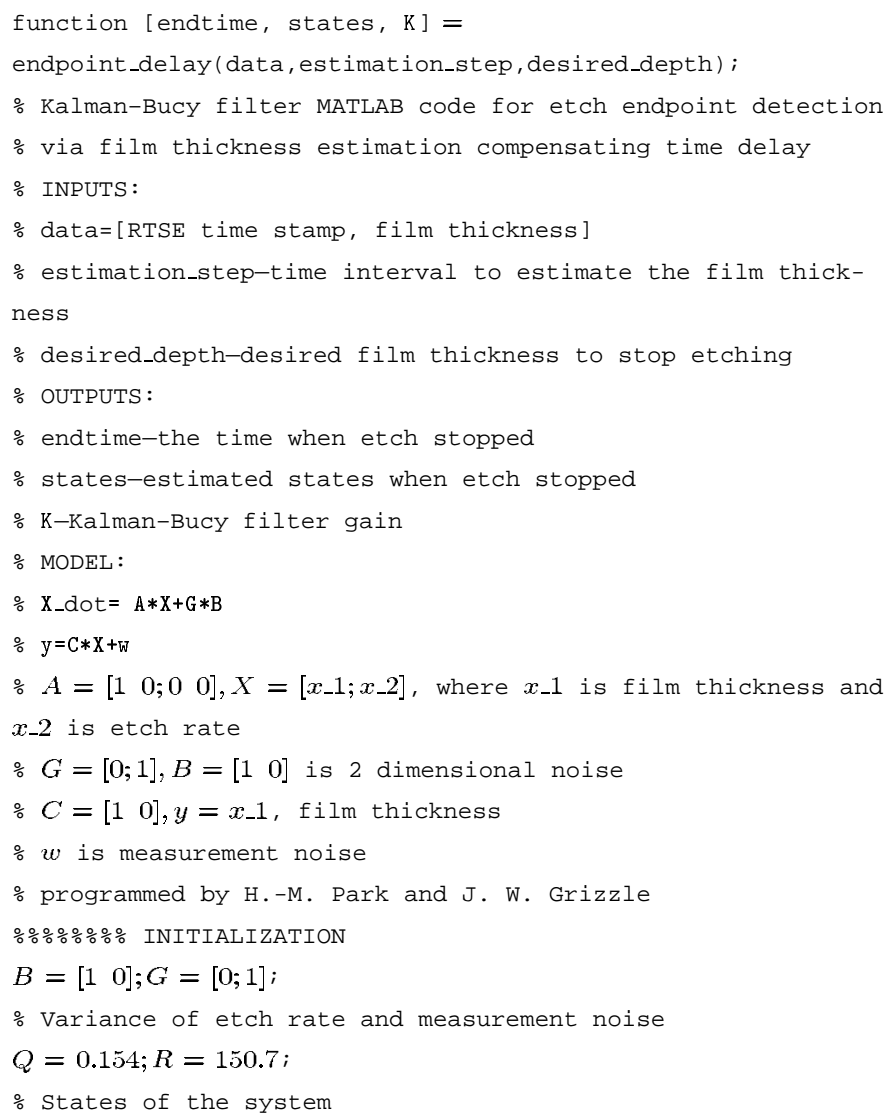




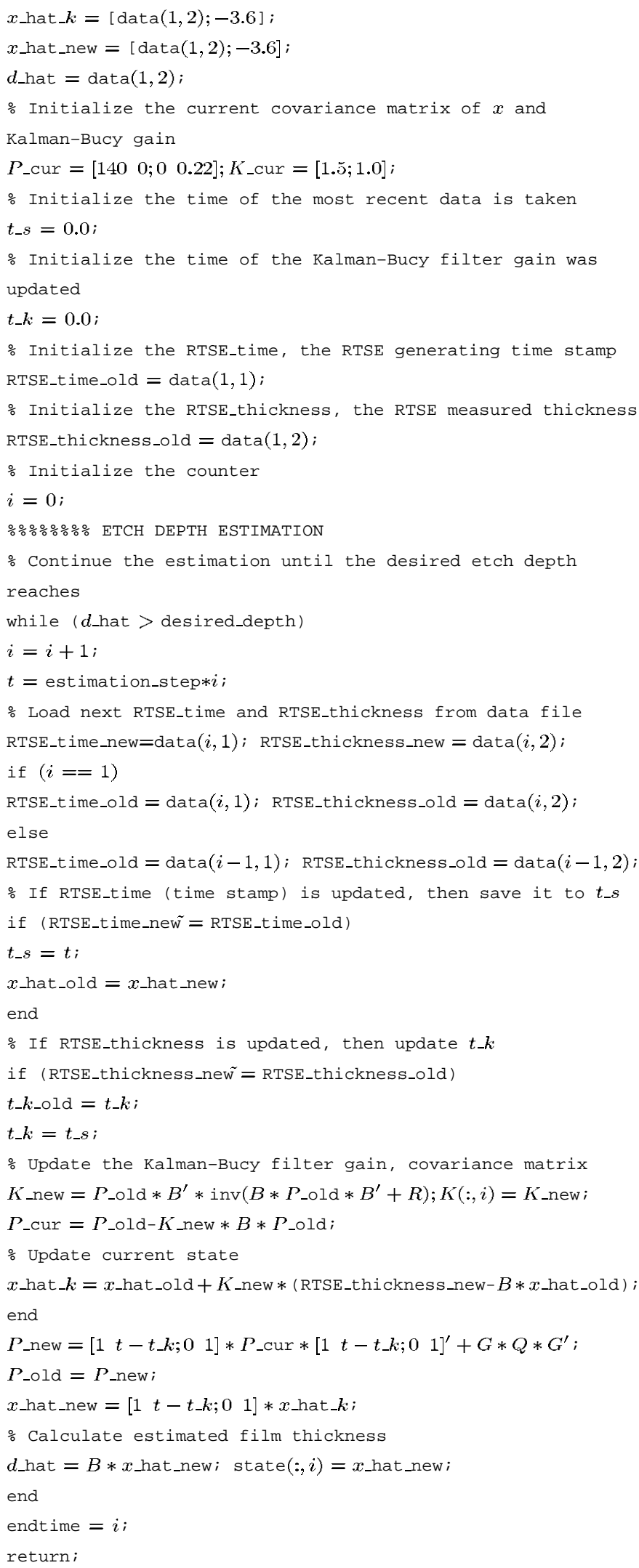

\section{ACKNOWLEDGMENT}

The authors sincerely thank T. Brock (University of Michigan) for help with the e-beam lithography.

\section{REFERENCES}

[1] T. D. Bestwick and G. S. Oehrlein, "Reactive ion etching of silicon using bromine containing plasmas," J. Vac. Sci. Technol., vol. A 8, no. 3, pp. 1696-1701, May/June 1990.

[2] L. Y. Tsou, "Highly selective reactive ion etching of polysilicon with hydrogen bromide," J. Electrochem. Soc., vol. 136, no. 10, pp. 3003-3006, Oct. 1989.

[3] R. J. Hoekstra, M. J. Grapperhaus, and M. J. Kushner, "Integrated plasma equipment model for polysilicon etch profiles in an inductively coupled plasma reator with subwafer and superwafer topography," $J$. Vac. Sci. Technol., vol. A 15, no. 4, pp. 1913-1921, July/Aug. 1997.

[4] P. D. Hanish, "Phenmenological Modeling of Reactive Ion Etching for Real-Time Feedback Control," Ph.D. thesis, Univ. of Mich., 1996.

[5] M. J. Kushner, "Advances in plasma equipment modeling," Solid State Technol., vol. 6, pp. 135-144, June 1996.

[6] C. Spanos, "Statistical process control in semiconductor manufacturing," Microelectron. Eng., vol. 10, no. 3-4, pp. 271-276, Feb. 1991.

[7] B. A. Rashap, M. E. Elta, H. Etemad, J. P. Fournier, J. S. Freudenberg, M. D. Giles, J. W. Grizzle, P. T. Kabamba, P. P. Khargonekar, S. Lafortune, J. R. Moyne, D. Teneketzis, and F. L. Terry Jr., "Contol of semiconductor manufacturing equipment: Real-time feedback control of a reactive ion etcher," IEEE Trans. Semiconduct. Manufact., vol. 8, pp. 286-297, Aug. 1995.

[8] T. H. Smith, S. Boning, J. Stefani, and S. W. Butler, "Run to run advanced process control of metal sputter deposition," IEEE Trans. Semiconduct. Manufact., vol. 11, pp. 276-284, May 1998.

[9] R. N. Castellano, "Profile control in plasma etching $\operatorname{sio}_{2}$," Solid State Technol., pp. 203-206, May 1984.

[10] R. H. Bruce, "Anisotropy control in dry etching," Solid State Technol., pp. 64-68, Oct. 1981

[11] P. Klimechy, D. Schweiger, and J. W. Grizzle, "Real-time data acquisition \& feedback control solution for industrial plasma reactors," presented at the Electrochem. Soc. Spring Meeting, Seattle, WA, 1999.

[12] J. N. Hilfiker and R. A. Synowicki, "Spectroscopic ellipsometry for process applications," Solid State Technol., vol. 39, no. 10, pp. 157-167, Oct. 1996

[13] S. W. Butler and J. A. Stefani, "Supervisory run-to-run control of polysilicon gate etch using in situ ellipsometry," IEEE Trans. Semiconduct. Manufact., vol. 7, pp. 193-201, May 1994.

[14] S. Maung, S. Bannerjee, D. Draheim, S. Henck, and S. W. Butler, "Integration of in situ spectral ellipsometry with mmst machine control," IEEE Tran. Semiconduct. Manufact., vol. 7, pp. 184-192, May 1994.

[15] R. A. Synowicki, "Spectroscopic ellipsometry characterization of indium tin oxide film microstructure and optical constants," Thin Solid Films, vol. 313-314, no. 1-2, pp. 394-397, Feb. 1997.

[16] H. Arimoto, S. Nakamura, S. Miyata, and K. Nakagawa, "Monitoring of sram gate patterns in KrF lithography by ellipsometry," IEEE Trans. Semiconduct. Manufact., vol. 12, pp. 166-169, May 1999.

[17] R. N. M. Azzam and N. M. Bashara, Ellipsometry and Polarized Light. Amsterdam, The Netherlands: North-Holland, 1987.

[18] C. J. Mogab and T. A. Shankoff, "Plasma etching of titanium for application to the patterning of ti-pd-au metallization," J. Electrochem. Soc. vol. 124, no. 11, pp. 1766-1771, Nov. 1977.

[19] D. Tennant, F. Klemens, T. Sorsch, F. Baumann, G. Timp, N. Layadi, A. Kornblit, B. J. Sapjeta, J. Rosamilia, T. Boone, B. Weir, and P. Silverman, "Gate technology for $70 \mathrm{~nm}$ metal-oxide-semiconductor fieldeffect transistor with ultrathin ( $<2 \mathrm{~nm}$ ) oxide," J. Vac. Sci. Technol., vol. B 15, no. 6, pp. 2799-2805, Nov./Dec. 1997.

[20] T. L. Vincent, P. P. Khargonekar, and F. L. Terry Jr., "Extended Kalman filtering-based method of processing reflectometry data for fast in-situ etch rate measurements," IEEE Trans. Semiconduct. Manufact., vol. 10, pp. 42-51, Feb. 1997.

[21] C. G. Galarza, P. P. Khargonekar, N. Layadi, T. L. Vincent, E. A. Rietman, and J. T. C. Lee, "New algorithm for real-time thin film thickness estimation given in situ multiwavelength ellipsometry using an extended Kalman filter," Thin Solid Films, vol. 313-314, no. 1-2, pp. 156-160, Feb. 1997

[22] A. H. Jazwinski, Stochastic Processes and Filtering Theory. New York: Academic press, 1970. vol. 64 of Mathematics in Science and Engineering.

[23] Y. Taur, Y. J. Mii, D. J. Frank, H. S. Wong, D. A. Buchanan, S. J. Wind, S. A. Rishton, G. A. Sai-Halasz, and E. J. Nowak, "CMOS scaling into the 21st century: $0.1 \mu \mathrm{m}$ and beyond," IBM J. Res. Develop., vol. 39 , no. 1/2, pp. 246-260, Jan./Mar. 1995

[24] M. Nakamura, K. Iizuka, and H. Yano, "Very high selective $n^{+}$poly-Si RIE with carbon elimination," Jpn. J. Appl. Phys., vol. 28, no. 10, pp. 2142-2146, Oct. 1989. 
[25] I. W. Rangelow and H. Löschner, "Reactive ion etching for microelectrical mechanical system fabrication," J. Vac. Sci. Technol., vol. B 13, no. 6, pp. 2394-2399, Nov./Dec. 1995.

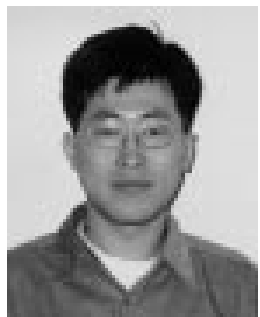

Hyun-Mog Park (S'97-M'00) received the B.S. degree in electronics engineering from the Korea University, Korea, in 1994 and M.S.E. and Ph.D. degrees in 1996 and 2000, respectively, all from the University of Michigan, Ann Arbor.

His doctoral research topic was real-time feedback control and fault detection in deep submicrometer etch process. He is currently a senior process engineer at Intel Components Research, Hillsboro, OR. His current research interests are fine feature patterning, ultra low-K dielectric etch, ash, and novel cleaning process development.

Dr. Park is a member of Tau Beta Pi and Eta Kappa Nu.

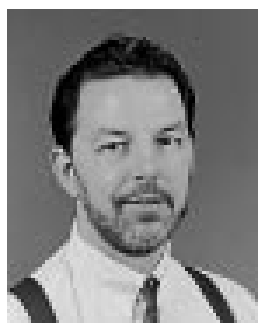

Dennis S. Grimard received the Ph.D. degree in electrical engineering from the University of Michigan at Ann Arbor in 1990.

After graduation, he accepted a position with IBM where he provided worldwide hardware, software and process solutions in support of IBM's 0.35and $0.5-\mu \mathrm{m}$ CMOS technologies. While at IBM, he was recognized for his technical achievements when IBM presented him a First Plateau Award (1995), a corporate Blue Chip Award (1995), an Outstanding Technical Achievement Award (1995) and a First Patent Award (1995). Currently, he is with the University of Michigan where he is a Research Scientist and Laboratory Manager for the Solid-State Electronics Laboratory. His major research interests lie in the field of RF metrology as it pertains to RIE process control. Since his doctoral work, he has co-authored numerous papers dealing with the theoretical and practical limitations of RF metrology and feed-forward control. He has been a consultant for the semiconductor industry since 1994, where he jointly holds ten patents on electrostatic chuck technology. He is also a joint author on another twenty patents currently under review by the U.S. patent office.

Dr. Grimard is a member of Tau Beta Pi, Eta Kappa Nu and has been an IBM $(1988,1989)$ and DeVlieg $(1986,1987)$ Fellow. He also received the Salisbury award from Worcester Polytechnic Institute in 1979.

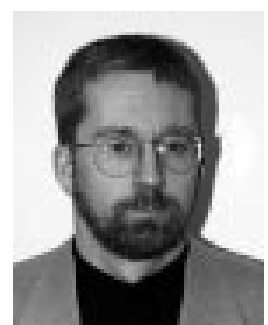

Jessy W. Grizzle (S'78-M'79-SM'90-F'97) received the Ph.D. degree in electrical engineering from the University of Texas at Austin in 1983.

Since September 1987, he has been with the University of Michigan, Ann Arbor, where he is a Professor of Electrical Engineering and Computer Science. He is a past Associate Editor of the IEEE TRANSACTIONS ON Automatic CONTROL and Systems \& Control Letters, and from 1997 to 1999 served on the IEEE Control System Society's Board of Governors. He has worked on a wide range of theoretical issues in nonlinear control and observer design, and is actively pursuing applications of system theory in the automotive industry and semiconductor manufacturing. Web:http://www.eecs.umich.edu/ grizzle/.

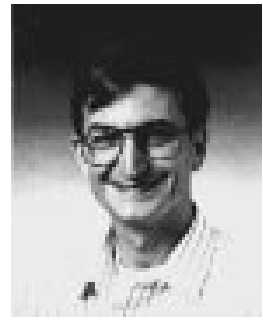

Fred L. Terry, Jr. (S'78-M'80-SM'99) received the B.S. and M.S. degrees in 1981 and the Ph.D. degree in 1985, all in electrical engineering from the Massachusetts Institute of Technology, Cambridge. His doctoral work involved the electronic properties of ammonia-annealed silicon dioxide films (nitrided oxides).

After completing his education, he joined the Department of Electrical Engineering and Computer Science at the University of Michigan, Ann Arbor, where he is currently an Associate Professor. His current research activities include nondestructive and in situ thin-film characterization techniques including spectroscopic ellipsometry and reflectometry, control of semiconductor processes and equipment, sensing systems for process control, plasma etch and deposition processes. He and his students have previously conducted research on low-temperature deposition of high quality insulators for MIS devices on $\mathrm{Si}$, InP, and GaAs, InP HFET device fabrication and physics, deep submicrometer etch processes, and processes for high- temperature Si MOS operation and for operation in ionizing radiation environments.

Dr. Terry is a member of the IEEE Electron Devices Society, the American Physical Society, the Electrochemical Society, the Metallurgical Society of the AIME, the Materials Research Society, and Sigma Xi. 